\title{
Counting on Inhibition and Rate-Dependent Excitation in the Auditory System
}

\author{
Christofer J. Edwards, Christopher J. Leary, and Gary J. Rose \\ University of Utah, Department of Biology, Salt Lake City, Utah 84112
}

The intervals between acoustic elements are important in audition. Although neurons have been recorded that show interval tuning, the underlying mechanisms are unclear. The anuran auditory system is well suited for addressing this problem. One class of midbrain neurons in anurans responds selectively over a narrow range of pulse-repetition rates (PRRs) and only after several sound pulses have occurred with the "correct" timing. This "interval-counting" process can be reset by a single incorrect interval. Here we show, from whole-cell patch recordings of midbrain neurons in vivo, that these computations result from interplay between inhibition and ratedependent excitation. An individual pulse or slowly repeated pulses elicited inhibition and subthreshold excitation. Excitation was markedly enhanced, however, when PRR was increased over a neuron-specific range. Spikes were produced when the enhanced excitation overcame the inhibition. Interval-number thresholds were positively correlated with the strength of inhibition and number of intervals required to augment the excitation. Accordingly, interval-number thresholds decreased when inhibition was attenuated by loading cells with cesium fluoride. The selectivity of these neurons for the interpulse interval, and therefore PRR, was related to the time course of excitatory events and the rate dependence of enhancement; for cells that were tuned to longer intervals, EPSPs were broader, and enhancement occurred at slower PRRs. The frequency tuning of the inhibition generally spanned that of the excitation, consistent with its role in temporal computation. These findings provide the first mechanistic understanding of interval selectivity and counting in the nervous system.

Key words: intracellular; torus semicircularis; temporal coding; amplitude modulation; inferior colliculus; anuran; auditory; acoustic; in vivo; interval; neuroethology; patch clamp

\section{Introduction}

Temporal intervals are defining components of music (Cooper and Meyer, 1960), as well as signals that are used for acoustic communication (Myrberg et al., 1978; Ehret, 1996; Diehl and Lindblom, 2004) and echolocation (Moss and Schnitzler, 1995). Although intervals can be represented by the timing of spikes in the sensory periphery, primary afferents respond to a variety of intervals and thus fail to code for particular intervals (Rose and Capranica, 1985). Neurons have been found in the CNS, however, that respond best when at least two acoustic elements are presented with the correct temporal spacing (Edwards et al., 2002) and order (Suga, 1989; Margoliash and Fortune, 1992). Although progress has been made in understanding the origin of selectivity for temporal features such as signal duration (Casseday et al., 1994, 2000; Covey et al., 1996) and temporal order (Lewicki, 1996; Mooney, 2000; Rosen and Mooney, 2003), little is known regarding the mechanisms that underlie interval selectivity.

Anuran amphibians (frogs and toads) are well suited for in-

Received June 20, 2007; revised Sept. 26, 2007; accepted 0ct. 2, 2007

This work was supported by a grant from the National Institutes of Health. We thank Y. Hanabusa, J. Callaway, and Shushruth for technical assistance and Dr. E. Brenowitz and T. Bryenton for help in collecting H. regilla.

Correspondence should be addressed to Dr. Gary J. Rose, Department of Biology, University of Utah, 257 South 1400 East Room 204, Salt Lake City, UT 84112. E-mail: rose@bioscience.utah.edu. DOI:10.1523/JNEUROSCI.2816-07.2007

Copyright $\odot 2007$ Society for Neuroscience $\quad 0270-6474 / 07 / 2713384-09 \$ 15.00 / 0$ vestigating mechanisms of interval selectivity. In anuran communication signals, intervals between successive sound pulses convey information about call type and species identity (Rose and Brenowitz, 1997, 2002; Gerhardt, 2001). A potential neural correlate of selective behavioral responses is found in the torus semicircularis (anuran inferior colliculus) where neurons show interval tuning (Alder and Rose, 1998; Edwards et al., 2002; Edwards and Rose, 2003). The most selective of these cells fail to respond to individual sound pulses, regardless of their duration, and respond only after a sufficient number of sequential pulses have been presented with optimal intervals. Remarkably, the intervalcounting process can be reset (set to zero) by a single interval that is too long (reset time) or, in some cases, too short (Edwards et al., 2002). This interval selectivity accounts for the sharp tuning of these cells to pulse-repetition rate (PRR). In addition, most neurons that have low interval-number thresholds (approximately one to three) respond to tone bursts of sufficient duration and hence are less temporally selective than those that require more intervals (Edwards and Rose, 2003).

The mechanisms that underlie this interval counting and selectivity are unknown. If the integration times were primarily determined by classical temporal summation, they should be accompanied by comparable reset times. Current evidence indicates, however, that reset times across cells are uncorrelated with and, in most cases, shorter than the integration times (number of intervals required to elicit spikes $\times$ interval duration). Also in- 


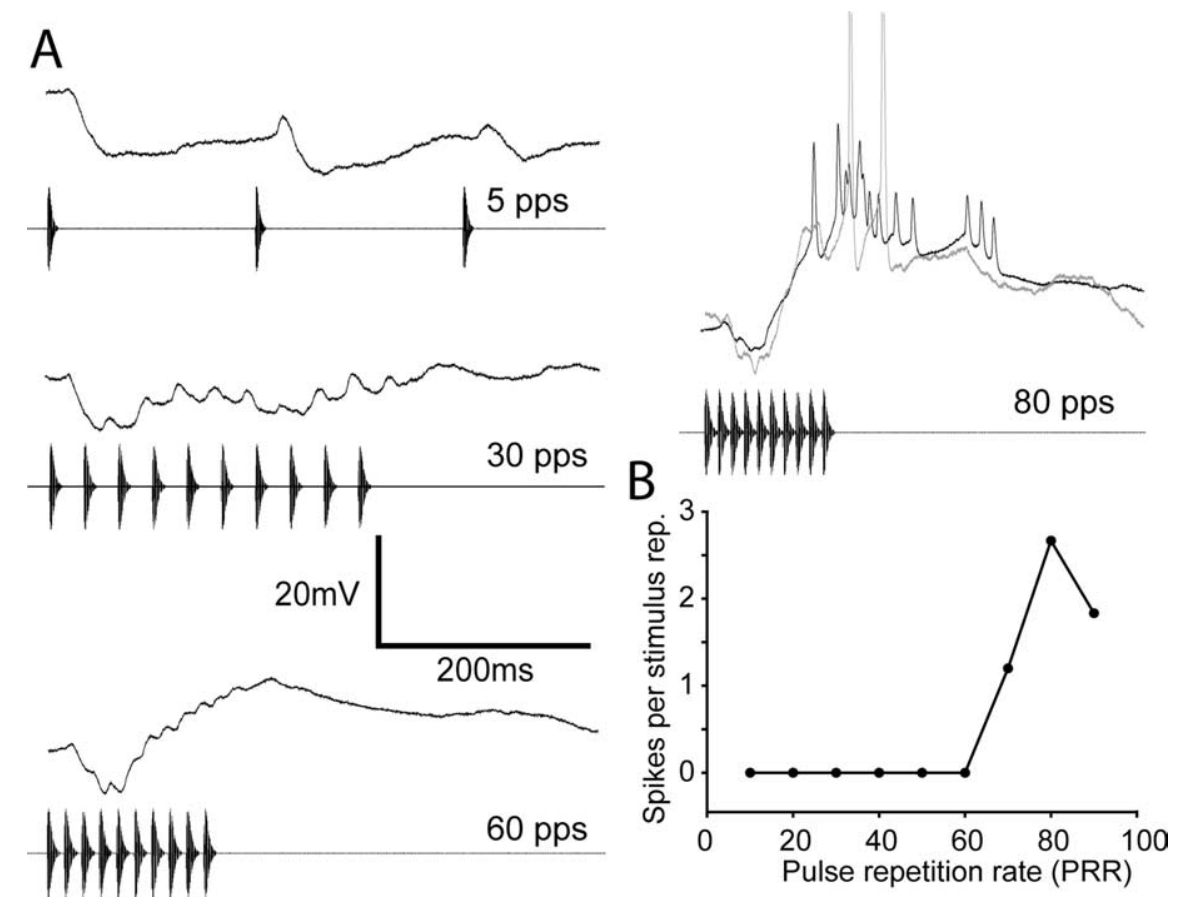

Figure 1. Intracellular recordings, in vivo, from an interval-counting neuron in the torus semicircularis of $H$. regilla. $\boldsymbol{A}$, Averaged responses to stimuli that consisted of 10 pulses, each 10 ms in duration, presented at rates of 5, 30,60, and 80 pps; at 5 pps, only the first three of the 10 pulses in the stimulus and responses are shown. Each black trace is an average of four to nine repetitions of the stimulus; the gray trace (top right) is a response to a single presentation of the $80 \mathrm{pps}$ stimulus. Sharp inflections on the top of the averaged trace at 80 pps represent action potential (spike) occurrences. The carrier frequency was $1600 \mathrm{~Hz}$, the BEF of the neuron. Stimulus amplitude was 68 $\mathrm{dBSPL}$, and the threshold of the cell was $56 \mathrm{dBSPL}$. The resting potential was $-66 \mathrm{mV}$. $\boldsymbol{B}$, Response [spikes per stimulus repetition (rep)] versus stimulus PRR for the same neuron.

consistent with the temporal summation model is the finding that these cells fail to respond to stimuli in which intervals are alternately shorter or longer than the optimal interval (Edwards et al., 2002); these stimuli have the same temporal pulse density as optimal-interval stimuli. Temporal summation alone therefore does not appear to account for the interval-counting process. Theoretically, however, interval selectivity could result from the integration of excitatory and inhibitory synaptic processes that differ in short-term synaptic plasticity (Buonomano, 2000). In this model, frequency-dependent depression of inhibition and facilitation of excitation results in preferential responses to repeated short intervals. We investigated the mechanisms that underlie the interval selectivity and interval-counting properties of midbrain neurons in anurans by recording intracellularly, in vivo, using the whole-cell patch method.

\section{Materials and Methods}

Recording procedures. Animals were prepared for recording according to methods published previously (Alder and Rose, 2000). Whole-cell patch (Ferster and Jagadeesh, 1992; Rose and Fortune, 1996) intracellular recordings were made, in vivo, from 247 auditory neurons in the torus semicircularis of 41 Rana pipiens and $30 \mathrm{Hyla}$ regilla; fifty-four of these were interval-counting cells. The advertisement calls of these two species have average PRRs of $\sim 15$ pulses/s (pps) and $90 \mathrm{pps}$, at $18^{\circ} \mathrm{C}$, respectively. This difference was exploited to determine whether intervalcounting neurons were specialized for processing advertisement call temporal structure; no such relationship was found.

"Whole-cell" recordings were made according to methods described in detail by Rose and Fortune (1996). Patch pipettes were constructed from borosilicate capillary glass $(1 \mathrm{~mm}$ outer diameter, $0.58 \mathrm{~mm}$ inner diameter; \#5960; A-M Systems, Everett, WA) using a Flaming-Browntype puller (model P-97; Sutter Instruments, Novato, CA). Electrodes were pulled to resistances between 15 and 25 $\mathrm{M} \Omega$. Electrode tips were back-filled with a solution ( $\mathrm{pH} 7.4$ ) consisting of (in $\mathrm{mm}$ ) 100 potassium gluconate, $2 \mathrm{KCl}, 1 \mathrm{MgCl}_{2}$, 5 EGTA, 10 HEPES, $20 \mathrm{KOH}$, and 43 biocytin to bring the final osmolarity to $\sim 285$ mOsm. Biocytin was replaced by mannitol in the solution used to fill pipette shanks. In other experiments, postsynaptic inhibition was attenuated by substituting cesium fluoride (CsF) for potassium gluconate in the recording pipettes (Nelson et al., 1994).

Seal resistances were typically $>2 \mathrm{G} \Omega$, and access resistances were typically $<50 \mathrm{M} \Omega$. For interval-counting neurons, resting potentials ranged from -54 to $-95 \mathrm{mV}$ (median, $-68 \mathrm{mV}$ ). Input resistances ranged from $181 \mathrm{M} \Omega$ to $1.3 \mathrm{G} \Omega$ (median, $558 \mathrm{M} \Omega$ ).

Patch pipettes had outside tip diameters of $\sim 1-2 \mu \mathrm{m}$. The pipette was advanced into the brain using an "inch-worm" microdrive (Burleigh Instruments, Fishers, NY) while applying positive pressure via a $30 \mathrm{cc}$ syringe to prevent clogging the tip. After reaching the recording location, the pipette was advanced in $1.5 \mu \mathrm{m}$ increments while maintaining slight positive pressure and passing $-0.1 \mathrm{nA}$ square-wave pulses $(500 \mathrm{~ms})$ to monitor resistance. Contact with a cell was indicated by a small increase $(10 \%)$ in the voltage change that resulted from this current injection. Subsequently, negative pressure was gradually applied to the pipette to increase the seal resistance to gigaohm levels. After a seal was formed, negative current (usually less than $-0.5 \mathrm{nA}$ ) was manually applied to rupture the patch and achieve an intracellular recording.

In the present study, the interaction between excitation and inhibition in controlling spike output was of primary interest. Because of this, along with space-clamp concerns, recordings were made in current-clamp mode.

Stimulus generation. Acoustic stimulus sets were constructed using Tucker Davis Technologies (Alachua, FL) System II hardware and custom-made software (Alder and Rose, 2000). Stimuli were presented free field in an audiometric room (Alder and Rose, 2000). The speaker was situated $0.5 \mathrm{~m}$ from the animal and contralateral to the recording site.

Neurophysiological procedures and measurements. The following procedures were used to record inhibition virtually isolated from excitation. Brief tone bursts (10-50 ms duration, $1 \mathrm{~ms}$ rise/fall times) and/or species-typical pulses were presented at rates less than one per second. These stimuli effectively elicited IPSPs but were highly ineffective in triggering excitation (supplemental Fig. 1, available at www.jneurosci.org as supplemental material). Second, we further minimized the influences of excitation by recording in positive current-clamp mode and depolarizing the neuron to near its excitatory reversal potential (see Fig. $8 \mathrm{~A}$ and supplemental Fig. $1 B$, available at www.jneurosci.org as supplemental material). It is important to emphasize that, under these conditions, the excitation was weak and brief to these stimuli, whereas the inhibition was prominent and longer lasting.

Depolarizations were identified as EPSPs, and not simply reversed IPSPs, on the basis of their capacity to trigger spikes when a sufficient number of intervals were presented. Because tone burst stimuli effectively elicited inhibition (see above), excitation could not be entirely "isolated" from the influences of inhibition. Inhibition was minimized by recording while the neuron was hyperpolarized to the inhibitory reversal potential (negative current-clamp recording). Nevertheless, the inhibitory conductance increase in response to a stimulus can shunt the excitation, thereby obscuring its full amplitude. To circumvent this problem, we measured EPSP amplitude at a point before the onset of the inhibition (i.e., where excitation and inhibition did not overlap). Although the peak of the EPSP generally could not be measured indepen- 
dent of inhibition, this procedure was sufficient for assessing the relative strength of excitation at various frequencies (i.e., determining the frequency tuning of the excitation). When excitation to a single tone burst was not easily detected, the frequency tuning of the excitation was determined by presenting several short bursts at the optimal PRR; although this is not a spectrally "pure" stimulus, at near-threshold sound levels it sufficed for characterizing the excitatory frequency tuning of the cell.

It is important to note that the primary objective of the frequency tuning analysis was to test the hypothesis that the frequency tuning of the inhibition spans that of the excitation. If the frequency tuning of inhibition and excitation are misaligned, attenuation of the excitation by inhibition should accentuate the misalignment (i.e., the shunting effects of inhibition are expected to bias the results against overlap).

The effects of attenuating inhibition with $\mathrm{CsF}$ were determined as follows. When spikes could be recorded after seal formation, response properties such as best excitatory frequency (BEF), threshold, best PRR, and interval-number threshold were determined before opening the cell. This procedure allowed us to quickly obtain baseline intracellular recordings of responses to critical stimuli once the patch was ruptured. In most cases, $\mathrm{Cs}^{+}$effects (e.g., broadening of action potentials, depolarization, and decrease in IPSP size) could be observed within 3-5 min of establishing an intracellular recording. In cases in which spikes could not be observed extracellularly, the neuron was first physiologically characterized while applying $\sim 0.01 \mathrm{nA}$ negative holding current to minimize $\mathrm{Cs}^{+}$ flow into the cell; the delivery of fluoride to the cell during this period did not appear to alter the size of IPSPs. Baseline interval-number thresholds were determined while this holding current was delivered and immediately after it was removed. In cases in which $\mathrm{Cs}^{+}$effects evolved slowly, loading was accelerated by passing $\sim 0.02 \mathrm{nA}$ positive current for $1-5$ min. Because $\mathrm{Cs}^{+}$loading was accompanied by depolarization of the neuron (Nelson et al., 1994), we recorded responses to sensory stimuli while injecting negative current to hold the cell near its normal resting potential. These recordings were compared with those made shortly after opening the patch (establishing a whole-cell intracellular recording). Changes in interval-number thresholds reported represent measurements made at a point where a clear $\mathrm{Cs}^{+}$action could be observed (i.e., spikes were broadened and stimulus-driven IPSPs were attenuated at least twofold). Because of differences across cells in the time course of $\mathrm{Cs}^{+}$action and duration of recording, we did not attempt to quantitatively analyze within-group differences in the magnitude of decreases in interval-number thresholds.

Threshold for eliciting enhancement of excitation was defined as the number of pulses, presented at the best PRR, required to elicit an EPSP that was at least $20 \%$ larger than the preceding EPSP; typically the increase was much more than $20 \%$ (median, $180 \%$ ). These enhanced EPSPs (see Fig. $2 B$ ) generally occur on a relatively stable background of inhibition. Accordingly, manipulating the influence of inhibition to a cell, through negative current clamp or $\mathrm{Cs}^{+}$, did not alter the enhancement threshold or time course of enhanced EPSPs once the inhibition had reached a steady-state level. Also, our preliminary analyses of conductances in these neurons [following the methods of Priebe and Ferster (2005)] indicate that the changes in the excitatory component $\left(g_{\mathrm{e}}\right)$ over time parallel those of the depolarizations (EPSPs); enhancement of EPSP amplitude and duration are mirrored by corresponding increases in the excitatory conductance. These data also establish that the depolarizations in response to successive stimulus pulses actually reflect changes in excitation to the cell, as opposed to changes in inhibition. The duration of enhanced EPSPs was measured at half-maximal amplitude (see Fig. $2 B$, horizontal arrow), defined as the midpoint between the membrane potential at the peak of the EPSP and that at the start of the EPSP. The stimulus had one less than the threshold number of pulses, delivered at the optimal PRR, and the EPSP to the last pulse was measured. Negative current-clamp-induced increases in the amplitude of EPSPs were not accompanied by changes in EPSP duration, suggesting that increased EPSP duration after a series of optimal intervals was not simply a byproduct of augmentation. Also, across neurons, the amplitude and duration of these depolarizations were not significantly correlated $\left(r^{2}=0.147 ; p=\right.$ $0.22 ; n=14$ ).

An index of inhibition strength for each neuron was determined in the
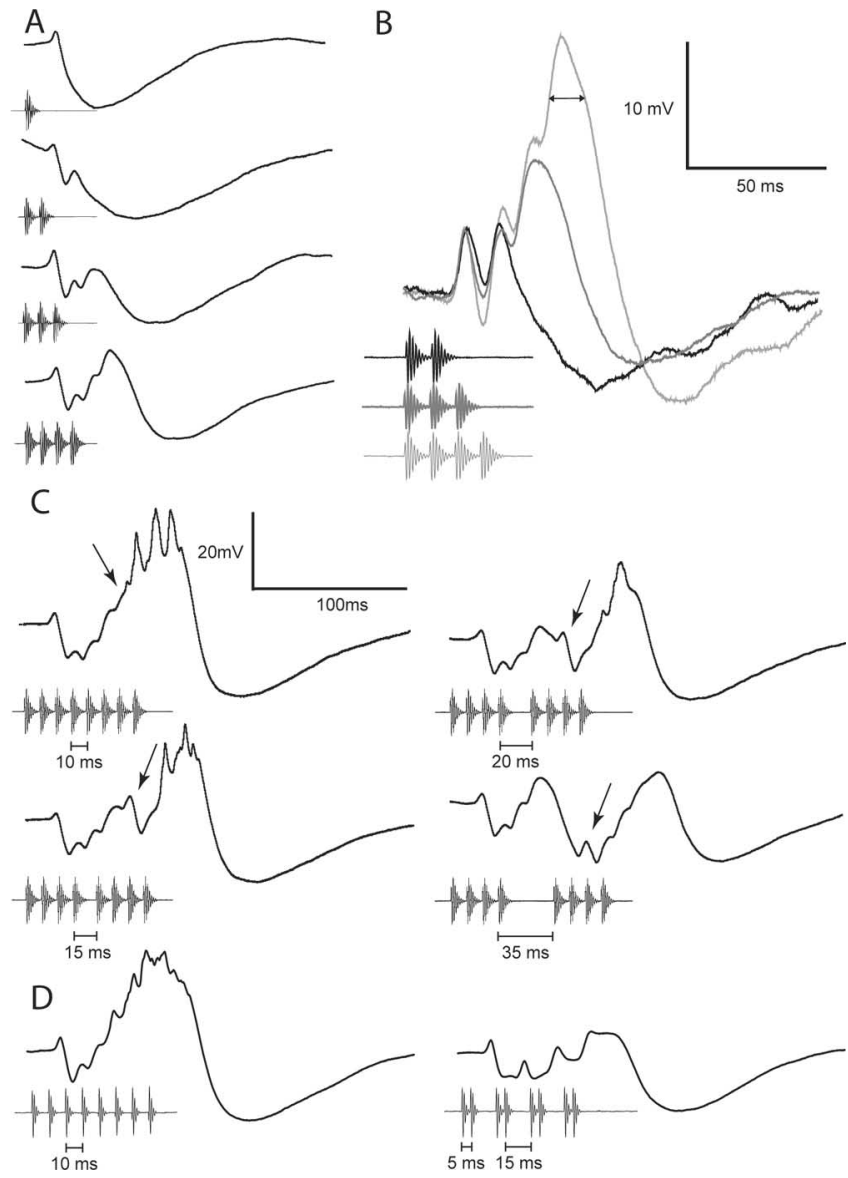

Figure 2. $\quad A-C$, Averaged intracellular recordings from an interval-counting neuron in R. pipiens to stimuli that differed in the number of pulses $(\boldsymbol{A}, \boldsymbol{B})$ or the duration of a middle interpulse interval of a pulse sequence ( $\boldsymbol{C}$. Responses shown in $\boldsymbol{B}$ are from recordings made while hyperpolarizing the neuron by $\sim 12 \mathrm{mV}$ (negative current-clamp mode). The horizontal arrow denotes EPSP duration at half-maximal amplitude; enhancement of EPSP amplitude is first observed to the third pulse. Arrows in Cindicate the EPSP elicited by the fifth pulse in each stimulus. The interval-counting process was reset by an interval of $35 \mathrm{~ms}$; hence, no spikes were elicited by the stimulus. Spikes were elicited by the other stimuli and appear as small peaks because of averaging. $\boldsymbol{D}$, Thirty-five spikes were elicited in 16 repetitions of the constant-interval stimulus (left) and one spike was elicited in 18 repetitions of the mixed-interval stimulus (right). Sound pulses were $5 \mathrm{~ms}$ in duration to prevent pulse overlap in the mixed-interval stimulus. The stimulus amplitude was $61 \mathrm{~dB}$ SPL in all panels. Stimulus repetitions for averages were 5-8(A), 4-7(B),8-13(C), and 16-18(D). Resting potential, $-70 \mathrm{mV}$; threshold, 49 $\mathrm{dBSPL} ; \mathrm{BEF}, 800 \mathrm{~Hz}$

following manner. The reversal potential for inhibition was ascertained while recording in negative current-clamp mode and presenting brief $(10-50 \mathrm{~ms})$ tone bursts. Positive current was then injected to depolarize the neuron to near the reversal potential of the excitation, and tone bursts (one burst per second) were delivered at an amplitude equal to the excitatory threshold of the neuron (lowest amplitude, at the BEF, at which spikes could be elicited by a stimulus of optimal PRR). The amplitude of IPSPs were measured and normalized according to the formula $\left(V_{c}-\right.$ $\left.V_{\mathrm{i}}\right) /\left(V_{\mathrm{c}}-V_{\mathrm{r}}\right)$, where $V_{\mathrm{c}}$ is the voltage of the membrane under positive current clamp, $V_{\mathrm{i}}$ is the voltage of the peak of the IPSP, and $V_{\mathrm{r}}$ is the reversal potential for inhibition. This calculation provided a measure of the effective inhibitory conductance. The influence of this conductance in opposing excitation depends on the driving force "behind" the inhibition, which was calculated as the difference between the threshold for spike initiation and the inhibitory reversal potential; a more negative inhibitory reversal potential resulted in a greater inhibitory current to oppose depolarization. An inhibition index was calculated by multiplying the former equation by this driving force $\left(D F_{\mathrm{I}}\right)$. This index reflects the influence of the inhibition in opposing excitation: $\left[\left(V_{\mathrm{c}}-V_{\mathrm{i}}\right) /\left(V_{\mathrm{c}}-V_{\mathrm{r}}\right)\right]$ $\times D F_{\mathrm{I}}$. 

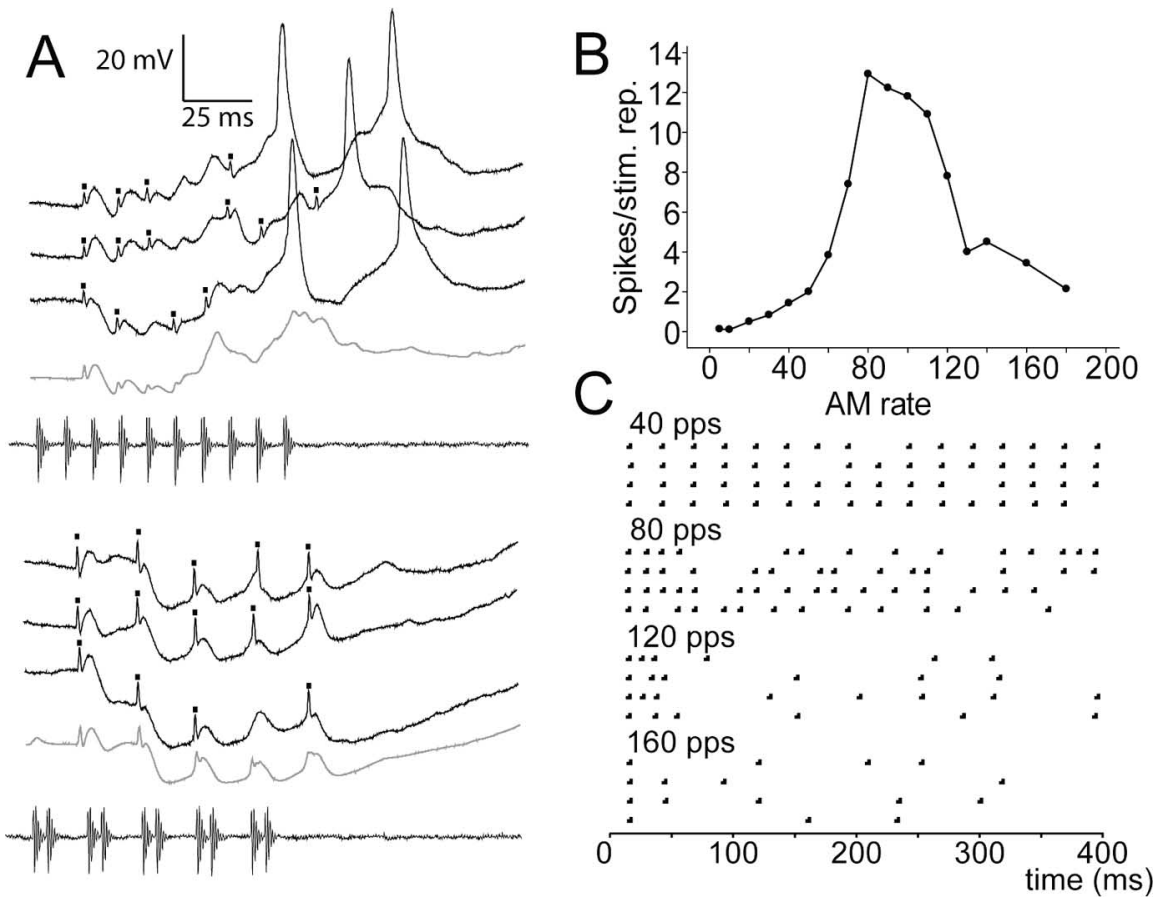

Figure 3. $A$, Responses to regular-interval ( $10 \mathrm{~ms}$; top) and mixed-interval ( 5 and $15 \mathrm{~ms}$; bottom) stimuli. The top three black traces in each panel are responses to single presentations. The small black squares mark the occurrences of spikes of a possible input to this neuron. The gray traces in each panel are averages of 61 repetitions for the regular-interval stimulus and 18 repetitions for the mixed-interval stimulus. $\boldsymbol{B}$, Spikes per stimulus repetition versus stimulus AM rate for the neuron in $\boldsymbol{A}$. All stimuli in $\boldsymbol{A}$ and $\boldsymbol{B}$ were presented at $46 \mathrm{~dB}$ SPL. The threshold for this neuron was $34 \mathrm{~dB}$ SPL. $\boldsymbol{C}$, Raster plots of the responses of the putative input to neuron in $\boldsymbol{A}$ to $40,80,120$, and 160 pps.

Statistical analyses. Multiple and linear regression analyses (JMP and Statview software; SAS Institute, Cary, NC) were used to determine the factors that predict interval-number thresholds and the best PRR of neurons.

\section{Results}

\section{Interval counting}

Intracellular recordings were made from 54 interval-counting neurons. A representative case (Fig. $1 A$ ) shows that the intervalcounting process and PRR selectivity result from interplay between inhibition and rate-dependent excitation. Species-typical pulses presented at slow rates (e.g., 5 pps) elicited primarily inhibition. At PRRs greater than $\sim 20$ pps, however, the amplitude of EPSPs increased with successive pulses. For 10 pulses, maximal response was reached at $\sim 80$ pps (Fig. 1). At this PRR, six pulses (five intervals) were required to elicit spikes.

The interplay between inhibition and rate-dependent excitation is best seen in the averaged responses of cells with prominent inhibition. For the exemplar shown in Figure $2 \mathrm{~A}$, an individual sound pulse elicited a small EPSP, which was followed by a larger and slower IPSP; recall that interval-counting neurons do not respond to an individual sound pulse. These slow hyperpolarizations are identified as inhibitory because they could be transformed into depolarizations when neurons were hyperpolarized sufficiently, and they were accompanied by increased conductance (data not shown). Additional pulses, delivered at $10 \mathrm{~ms}$ interpulse intervals, elicited additional EPSPs that, starting with the third, were enhanced in amplitude and duration. With four pulses, this augmentation of the excitation, along with temporal summation, resulted in a net depolarization of $\sim 7 \mathrm{mV}$. Four intervals (five pulses) were required for eliciting spikes. These additional pulses also extended the time course of the inhibition, as seen in the prominent hyperpolarization that follows the last EPSP. During this IPSP, the cell was hyperpolarized to -81 $\mathrm{mV}$ compared with $-78 \mathrm{mV}$ after a single pulse. These data suggest that little, if any, depression of the inhibition occurred in response to a series of optimal intervals. Across such cases, in which the time course of inhibition exceeded that of the excitation, the magnitude of the inhibition to a series of intervals did not differ significantly from that to a single pulse (IPSP ${ }_{\text {last }} /$ IPSP $_{\text {first }}=0.97 ; t_{(7)}=0.33 ; p=0.75$ ). However, in cases in which the time course of excitation to a series of intervals exceeded that of the inhibition, we could not accurately determine the time course of the inhibition. We therefore cannot rule out the possibility that rate-dependent depression of inhibition contributed to the interval-counting properties in some neurons.

To minimize the influence of inhibition and to assess the role that voltagedependent processes might play in enhancement with successive pulses, we also recorded the responses of this same cell while hyperpolarizing it by $\sim 12 \mathrm{mV}$ (i.e., negative current-clamp recording) (Fig. $2 B)$. The EPSPs in response to a pair of pulses (black trace) were comparable in amplitude. As was seen in the recordings without current clamp, presenting three or four pulses resulted in additional depolarizations that were larger and more sustained. These data suggest that the enhanced depolarizations in response to successive stimulus pulses actually reflect changes in excitation to the cell, as opposed to changes in inhibition. These results, which are representative of the cells recorded in this study, also suggest that postsynaptic voltage-dependent conductances are not responsible for the enhancement of excitation.

Can the interplay between inhibition and rate-dependent excitation account for the rapid resetting of the interval-counting process by a single incorrect interval? We investigated the basis of this resetting process by presenting two sets of optimal intervals that were each one shy of threshold and separated by an interval of varying dimensions (Fig. $2 C$ ). The reset time was determined by increasing the duration of the middle interval until spikes were no longer elicited by the second set of pulses. As expected for this exemplar neuron, a sequence of seven optimal $(10 \mathrm{~ms})$ intervals elicited enhanced excitation that overcame the inhibition (Fig. $2 C$ ) and evoked spikes (2.5 spikes per stimulus presentation; SE, 0.23 ). Remarkably, increasing the middle (fourth) interval from $10 \mathrm{~ms}$ to 15 or $20 \mathrm{~ms}$ reduced the response to 1.5 (SE, 0.31 ) and 0.9 (SE, 0.14) spikes per stimulus presentation, respectively. This decrease stemmed from the continued action of the inhibition and a reduction in the excitation. For the $20 \mathrm{~ms}$ interval, the depolarization (arrow) in response to the fifth pulse (i.e., the first pulse of the second set) is comparable in amplitude to that in response to the first pulse. The interval-counting process was reset (no spikes occurred on at least $75 \%$ of the presentations) by an interval of $\geq 35 \mathrm{~ms}$. For this stimulus condition, the inhibition elicited by the first set of pulses hyperpolarized the neuron by $\sim 15 \mathrm{mV}$, relative to resting potential. 


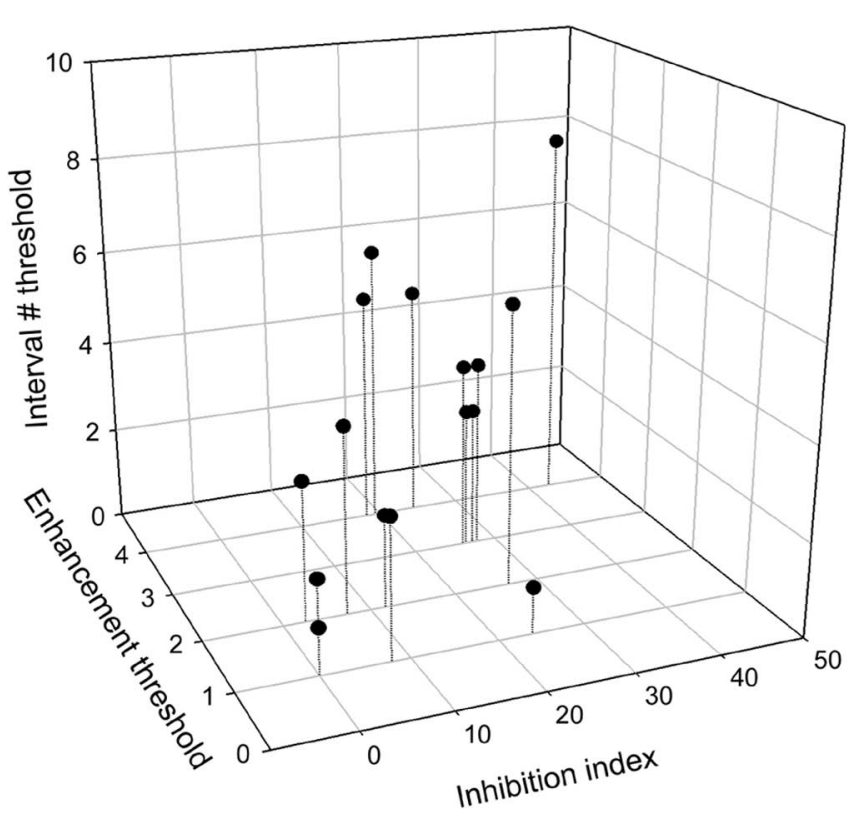

Figure 4. Interval-number threshold versus inhibition versus the number of pulses required for enhancement of excitation (enhancement threshold) for 16 neurons. The interval-number threshold was defined as the number of intervals, holding the interpulse interval constant at the optimal value, required to elicit one or more spikes on at least $50 \%$ of the stimulus presentations. The influence of inhibition in each case was quantified by computing an index that encompassed the magnitude of the inhibitory conductance and the driving force (see Materials and Methods).

Previous work showed that interval-counting neurons respond poorly, if at all, to stimuli that consist of intervals that are alternately shorter or longer than the optimal interval (Edwards et al., 2002). To provide insight into why mixed-interval stimuli are ineffective, we also recorded from this same neuron (Fig. 2D) while presenting stimuli in which eight sound pulses, each $5 \mathrm{~ms}$ in duration, were delivered with intervals that were either $10 \mathrm{~ms}$ (left) or alternated between 5 and $15 \mathrm{~ms}$ (right); thus, these stimuli had the same average PRR (100 pps). The responses of this neuron to mixed-interval stimuli are representative of those for three of the four interval-counting cells that were tested with this type of stimulus regimen. The sequence of regular intervals elicited fluctuations of the membrane potential (i.e., hyperpolarizations and depolarizations) that were highly similar to those seen for the corresponding sequence of $10 \mathrm{~ms}$ pulses (Fig. 2C); thirtysix spikes occurred in response to 16 presentations. For the mixed-interval stimulus, the first pair of pulses elicited a small depolarization (EPSP) and a larger hyperpolarization (IPSP), comparable to the response to the first pulse in the regularinterval stimulus. Some enhancement of EPSP amplitude can be seen in the responses to the third and fourth pairs of pulses; however, the depolarizations were insufficient for eliciting spikes on 17 of the 18 presentations of this stimulus. Some enhancement with intervals of $15 \mathrm{~ms}$ is expected because regular-interval stimuli of 60-70 pps also elicited enhancement but rarely triggered spikes. The fourth neuron that was tested with this stimulus regimen showed a similar pattern of hyperpolarizations and depolarizations for a regular-interval stimulus, but relatively little enhancement of EPSP amplitude was evident across responses to pairs of pulses in the mixed-interval stimulus (Fig. 3A). Inhibition also was elicited by the mixed-interval stimulus, and resulted in a hyperpolarization of $\sim 11 \mathrm{mV}$ between EPSPs. As in the previous case (Fig. 2D), EPSPs to the first pair of pulses in the

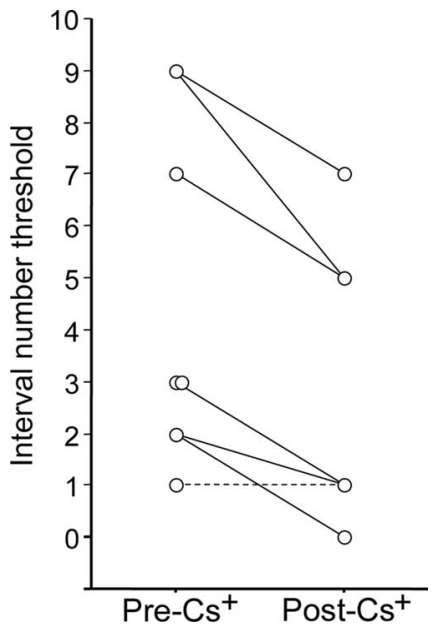

Figure 5. Interval-number thresholds of eight neurons before (Pre) and following (Post) attenuating inhibition. CSF was substituted for potassium gluconate in the recording pipette. Inhibition was attenuated after passing positive current to load the neuron with $\mathrm{Cs}^{+}$. The dashed line indicates data for the single interval-counting neuron that lacked stimulus-driven inhibition.

mixed-interval stimulus were similar in amplitude $(5 \mathrm{mV})$ and timing to those in response to the (single) first pulse of the regular-interval stimulus (Fig. $3 A$ ). This result suggests that excitatory afferents to this cell spiked to only the first pulse of each pair. Serendipitously, small spikes could be seen (marked by dots) that were triggered by stimulus pulses and may represent an afferent to this cell. This supposition is supported by the finding that an EPSP followed each spontaneous (i.e., not stimulus driven) small spike. For the mixed-interval stimulus, each pair of pulses triggered a single small spike (i.e., spikes code an interval of $20 \mathrm{~ms}$ ). The latency of the small spike from the onset of the first pulse in either the regular- or mixed-interval stimulus was $\sim 18$ $\mathrm{ms}$, also consistent with the notion that afferents to this cell spike to just the initial pulse of each pair. Together, these results suggest that the $5 \mathrm{~ms}$ intervals were not coded by a suitably timed pair of afferent spikes. The failure of afferents to represent repeated short intervals in the timing of their spikes may underlie the decrease in spike output of this neuron for PRRs above the best rate (Fig. $3 B$ ). In support of this notion, the putative afferent to this cell produced spikes in response to at least the first three to four pulses when presented at rates of 40 and 80 pps but not to rates above $\sim 100$ pps (Fig. 3C); at 160 pps, only the first pulse was reliably coded by a spike.

The recordings shown in Figures 1-3 suggest that interplay between inhibition and frequency-dependent excitation plays an important role in governing the interval-counting properties of a neuron. Accordingly, across all cells, interval-number thresholds were positively correlated with the enhancement threshold (the number of intervals required to enhance excitation; $F_{(1,14)}=$ $\left.18.42 ; p=0.001 ; r^{2}=0.568\right)$ and our index of inhibition (see Materials and Methods; $\left.F_{(1,14)}=9.13 ; p=0.009 ; r^{2}=0.395\right)$. That is, neurons that required many intervals to respond (spike) had prominent inhibition and showed enhancement of excitation only after many intervals; neurons that responded after a single interval tended to have little or no inhibition (Fig. 4). Correspondingly, the enhancement threshold and inhibition index were positively correlated $\left(F_{(1,14)}=10.89 ; p=0.005 ; r^{2}=0.44\right)$. Together, these two parameters account for nearly $60 \%$ of the variation in interval-number thresholds across neurons. Interval-number thresholds were not significantly correlated 


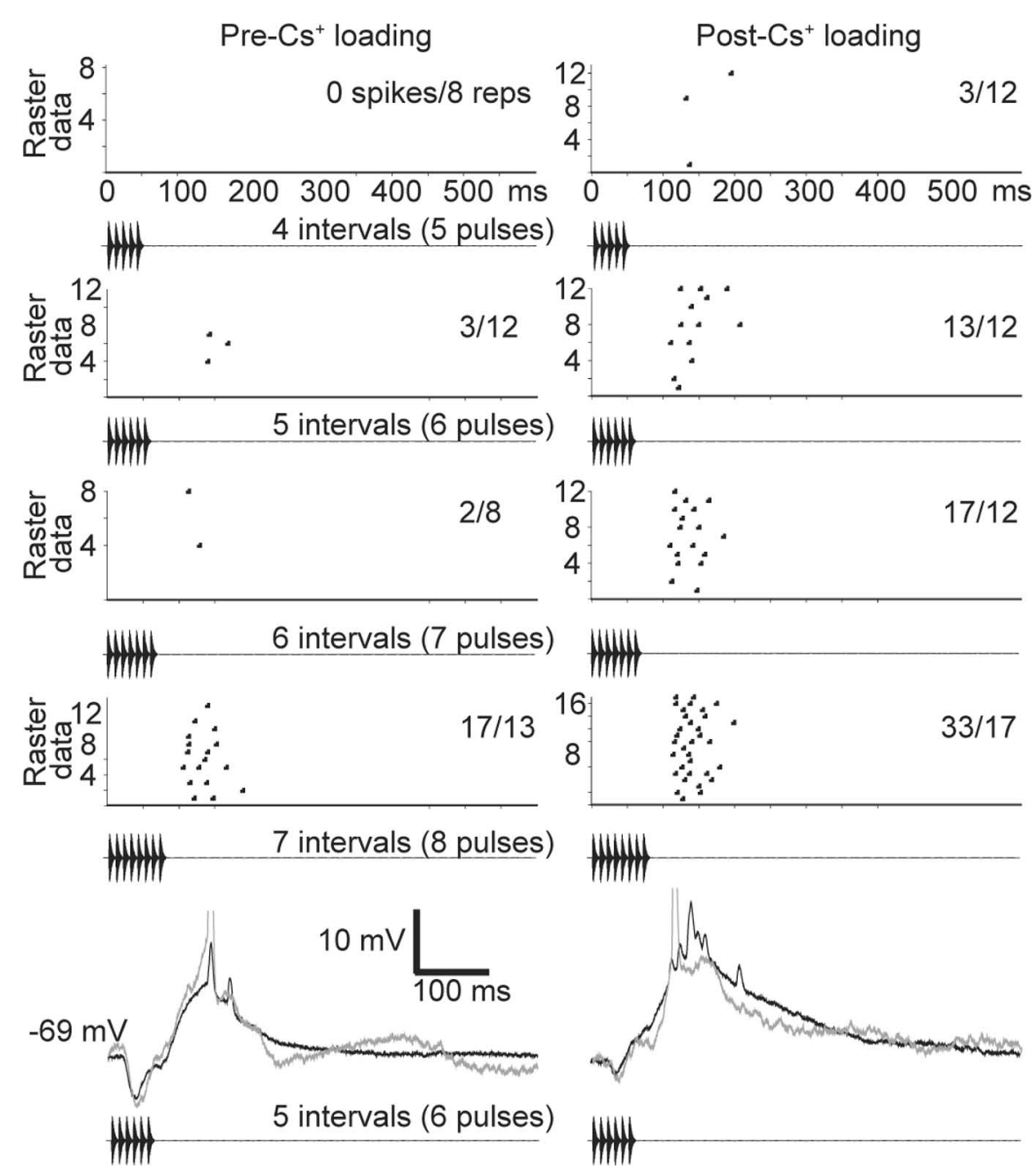

Figure 6. Raster plots of spike occurrences (dots) in response to stimuli that consisted of $5-8$ pulses, 100 pulses/s, before (left) and while (right) inhibition was attenuated by $\mathrm{Cs}^{+}$. Traces (bottom) show averaged (black) and unaveraged (gray) intracellular recordings (whole-cell patch method) from this neuron, recorded in $R$. pipiens, in response to stimuli consisting of six pulses. BEF, $1500 \mathrm{~Hz}$; threshold, $36 \mathrm{~dB} \mathrm{SPL}$; stimulus amplitude, $45 \mathrm{~dB}$ SPL.

with differences between spike initiation threshold and resting potential ( $p=0.65 ; n=15)$ nor with IPSP duration, measured at half-maximum amplitude $(p=0.61 ; n=13)$.

The positive correlation between interval-number thresholds and inhibition suggested that reducing inhibition should decrease the interval-number threshold of a cell. We tested this hypothesis by loading eight cells with $\mathrm{Cs}^{+}$to attenuate inhibition. The interval-number thresholds of all but one of the neurons were decreased by this treatment $\left(t_{(8)}=4.7 ; p=0.002\right.$ ) (Fig. $5)$; the single exception (dotted line) was a cell that showed no inhibition and responded after a single interval. An exemplar (Fig. 6), had a pre-Cs ${ }^{+}$threshold of seven intervals (left panels); few or no spikes were elicited by stimuli that had four to six intervals (five to seven pulses). The averaged subthreshold response to six pulses (Fig. 6, bottom) shows inhibition, resulting in a hyperpolarization of $\sim 7 \mathrm{mV}$, followed by depolarization and spiking. While the cell was progressively loaded with $\mathrm{Cs}^{+}$, there was a concomitant decrease in the magnitude of the hyperpolarization and increase in the number of spikes elicited by stimuli that consisted of four to six intervals (five to seven pulses) (Fig. 6, right panels).

\section{PRR selectivity}

Interval-counting neurons respond selectively over a particular range of PRRs. The PRR that elicited maximal response varied considerably across interval-counting neurons and even included cases that showed selectivity for rather low PRRs. An exemplar (Fig. 7A) had a best PRR (i.e., the PRR that elicited the most spikes per stimulus presentation) of $\sim 30$ pps. This cell showed enhancement of excitation even to 5 pps. What factors account for this variation in best PRR? Across neurons, the best PRR was negatively correlated with the duration of enhanced EPSPs, measured at half-maximal amplitude (Fig. $2 B)\left(F_{(1,12)}\right.$ $\left.=21.1 ; p<0.001 ; r^{2}=0.64\right)$, and positively correlated with the lowest PRR at which enhancement of excitation was first observed $\left(F_{(1,12)}=15.4 ; p=0.002\right.$; $\left.r^{2}=0.56\right)$. That is, neurons that were tuned to slow PRRs had longer-duration EPSPs and enhancement of excitation started at lower PRRs relative to those that were tuned to faster rates (Fig. $7 B$ ). A multiple regression analysis indicated that these two variables explained $79.7 \%$ of the variation in best PRR $\left(F_{(2,11)}=\right.$ $\left.21.6 ; p<0.001 ; r^{2}=0.797\right)$; inclusion of excitation enhancement thresholds (number of intervals required for enhancement), which alone were marginally correlated with best PRR $\left(F_{(1,15)}=\right.$ $\left.3.8 ; p=0.07 ; r^{2}=0.21\right)$, did not account for additional variation. Inhibition, although a predictor of interval-number thresholds, was not correlated with the best PRR ( $p=0.97 ; n=16)$. EPSP duration was important in determining the PRR at which sufficient temporal summation of enhanced excitation occurred. EPSP duration was unrelated to the time constants of these cells ( $p=0.41 ; n=8)$ and appears to be primarily governed by the time courses of excitatory conductances.

\section{Frequency tuning of excitation and inhibition}

These findings suggest that inhibition plays a critical role in performing temporal computations (Casseday et al., 1994; Wehr and Zador, 2003; Zhang et al., 2003). Inhibition might also, however, shape the frequency (spectral) selectivity of these neurons. Extracellular recording studies of the frequency tuning properties of central auditory neurons have suggested that lateral inhibition (generating inhibitory flanking regions to the frequency-tuning functions) is common in the superior olivary nucleus and inferior colliculus of anurans (Hall, 1999; Zheng and Hall, 2000). If the primary function of inhibition in interval-counting cells is to perform temporal computations, the frequency bandwidth of the inhibition should span that of the excitation. Alternatively, if the inhibition is "lateral," the frequency tuning of inhibition will be offset from that of the excitation (i.e., misaligned). This former hypothesis is supported by recordings shown in Figure $8 \mathrm{~A}$. In this case, the frequency tuning of the inhibition (red) and excitation 

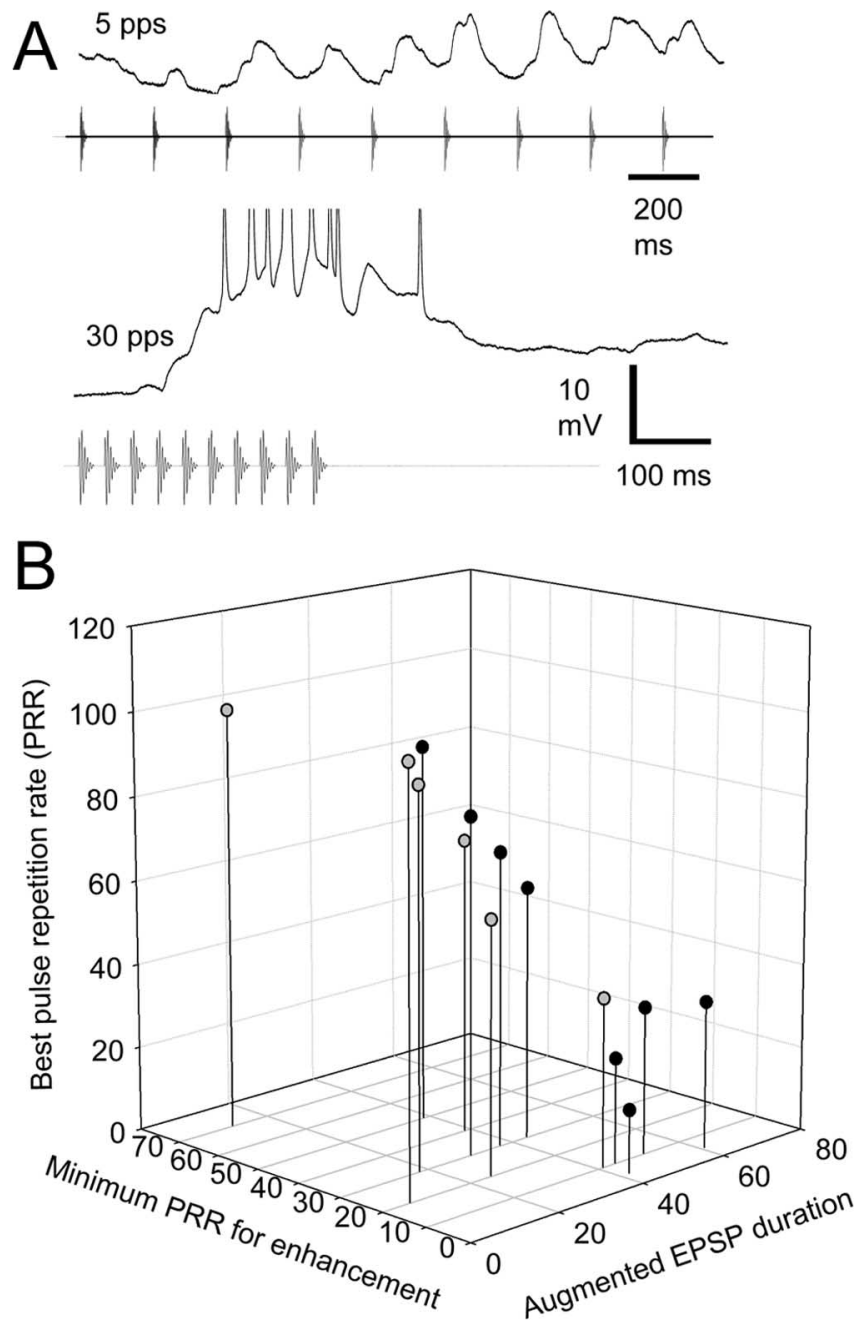

Figure 7. A, Intracellular recordings from a neuron in $H$. regilla that was tuned to $30 \mathrm{pps}$. Averaged traces of responses to $5 \mathrm{pps}$ (top) and $30 \mathrm{pps}$ (bottom). Each trace was an average of four repetitions of the stimulus ( $80 \mathrm{~dB} \mathrm{SPL}$ ). BEF, $280 \mathrm{~Hz}$; threshold, $71 \mathrm{~dB} \mathrm{SPL}$. $\boldsymbol{B}$, Relationship between the PRR that elicited maximal response, the slowest PRR that was effective for activating enhancement of excitation (enhancement threshold), and the duration of enhanced EPSPS.

(green) were mostly congruent, peaking in the $1.6-2.0 \mathrm{kHz}$ range. For a stimulus amplitude of $70 \mathrm{~dB}$ sound pressure level (SPL), the threshold for eliciting spikes, excitation, and inhibition extended to $\sim 1.1 \mathrm{kHz}$ and $800 \mathrm{~Hz}$, respectively. This overlap and general congruence of excitatory and inhibitory frequency tuning was also observed when the stimulus amplitude was reduced to $58 \mathrm{~dB}$ SPL (fourfold reduction). The broad tuning to frequencies near $2 \mathrm{kHz}$ indicates that this neuron received input of the basilar papilla type; anurans have two inner ear organs for detecting airborne sound, the amphibian papilla and basilar papilla (Lewis and Lombard, 1988). Because primary afferents that innervate the basilar papilla are rather uniformly tuned (i.e., have similar BEFs and breadth of tuning), it could be argued that the congruence of excitatory and inhibitory frequency tuning is not surprising.

We therefore further examined the congruence of the excitatory and inhibitory frequency tuning, for interval-counting neurons that had lower BEFs (i.e., frequencies represented by the amphibian papilla). Primary afferents that innervate the amphibian papilla exhibit a diversity of BEFs and bandwidths. Nevertheless, the frequency tuning of the inhibition was broader than $(z=$
$-2.76 ; n=13 ; p=0.006$, Wilcoxon signed rank test) and, in all but one case, overlapped extensively with that of the excitation (Fig. $8 B, C$ ). This finding also supports the role of inhibition in temporal pattern analysis (Casseday et al., 1994; Wehr and Zador, 2003; Zhang et al., 2003), rather than solely for sharpening frequency tuning (lateral inhibition).

\section{Discussion}

These data (the first whole-cell patch recordings, in vivo, from the anuran auditory system) show that the interval-counting properties of midbrain auditory neurons result, in large part, from interplay between inhibition and rate-dependent excitation. These findings therefore generally support the model of interval selectivity proposed by Buonomano (2000) and support the notion that complex computations can, to a large extent, result from the integration of synaptic input by single neurons (Herz et al., 2006).

In this integration process, inhibition opposes excitation and increases the number of intervals required for eliciting spikes. Enhancement of excitation opposes inhibition and therefore plays a role in setting interval-number thresholds. Together, these two parameters account for nearly $60 \%$ of the variation in interval-number thresholds across neurons. Rate-dependent depression of inhibition, a component of the interval selectivity model proposed by Buonomano (2000), does not appear to contribute to interval counting and selectivity in neurons that had relatively prolonged inhibition. This conclusion is supported by the finding that the hyperpolarization after a series of optimal intervals was generally as large as that after a single pulse. This persistent inhibition contributed to the resetting of the intervalcounting process. Nevertheless, the possibility that this form of plasticity may contribute to the interval selectivity and counting properties of some cells cannot be ruled out at this time and may account for some of the unexplained variance in interval-number thresholds.

Attenuating inhibition by loading neurons with $\mathrm{Cs}^{+}$directly supports the role of inhibition in determining interval-number thresholds. These findings also suggest that $\mathrm{K}^{+}$channels are responsible for this inhibition. Additional work is needed to identify the particular subtype (s) of $\mathrm{K}^{+}$channels that is involved. One possibility is that G-protein-coupled, inward rectifying potassium channels mediate the inhibition (Schmidt and Perkel, 1998). These channels have been shown to underlie inhibition in song-selective neurons of the avian nucleus HVC (Rosen and Mooney, 2003). Future studies could use blockers of these channels (e.g., GDP $\beta S$ ) (Andrade et al., 1986) to determine whether they underlie the inhibitory conductances in interval-counting neurons.

We have also shown that the best PRR for each neuron was primarily determined by the rate dependence of excitation enhancement and the time course of EPSPs; enhancement occurred at faster PRRs, and EPSP duration was shorter for neurons that were tuned to faster PRRs. Because interval-counting neurons are similarly tuned to PRR and rate of amplitude modulation (AM), these findings should be relevant to understanding mechanisms that underlie bandpass selectivity for AM rate, which has been observed across a wide range of taxa (Rose, 1986; Pollack, 2001). Another class of AM bandpass neurons shows strong selectivity for slow PRRs (Alder and Rose, 2000). Interplay between excitation and inhibition also appears to underlie this type of temporal selectivity in the anuran inferior colliculus (Edwards et al., in prep.).

Two lines of evidence indicate that the enhancement does not 


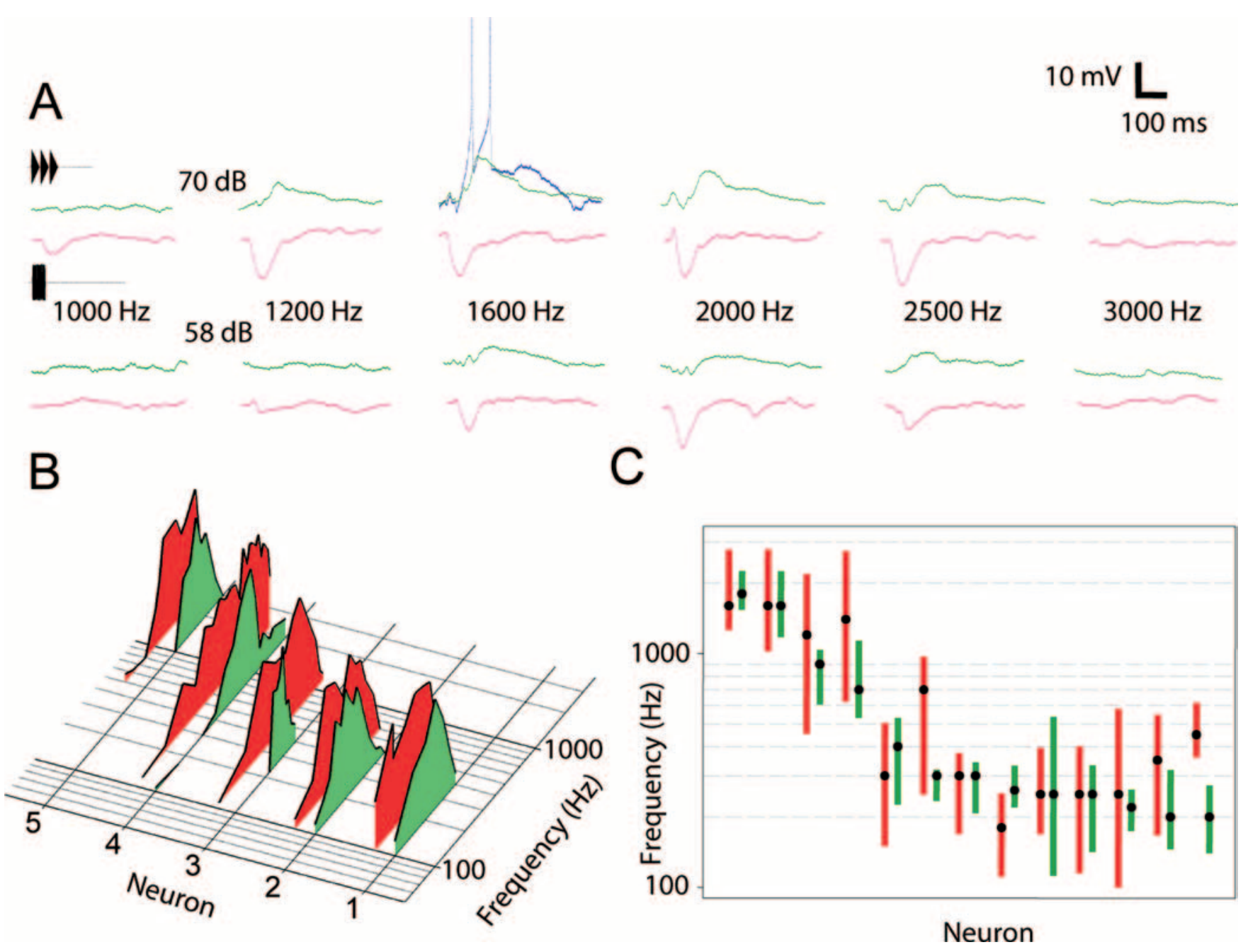

Figure 8. Frequency tuning of excitation (green) and inhibition (red) for interval-counting neurons. A, Averaged responses of a single neuron in $H$. regilla to stimuli with carrier frequencies and amplitudes (dB SPL) shown. The resting potential was $-65 \mathrm{mV}$. Recordings of inhibition or excitation were made while depolarizing or hyperpolarizing the neuron by $\sim 14$ and $4 \mathrm{mV}$, respectively. $B$, Strength of excitation and inhibition versus frequency for five neurons. Both the excitation and inhibition strengths were measured at stimulus amplitudes equal to the threshold sound level for spiking (decibels) for each neuron (see Materials and Methods). C, Range of frequencies where the strength of excitation (green) or inhibition (red) was at least half their respective maxima (derived from curves as in $\boldsymbol{B}$ ).

result from postsynaptic, voltage-dependent changes in depolarizing conductances. First, injecting current pulses at a rate equal to the best PRR for a cell resulted in temporal summation of depolarizations, without amplification of depolarizations to successive pulses (see supplemental material, available at www. jneurosci.org as supplemental material). Second, hyperpolarization does not abolish the enhancement. The rapid resetting of excitation strength by a single long interval would appear to rule out classical presynaptic facilitation (Atluri and Regehr, 1996; Zucker, 1999; Dittman et al., 2000) as a mechanism. Residual calcium levels, which are thought to underlie presynaptic facilitation, remain elevated during intervals of the dimensions that reset the enhancement in our cells. However, under conditions of strong buffering of residual $\mathrm{Ca}^{+2}$ at small presynaptic terminals, increases in $\mathrm{Ca}^{+2}$ levels are more transient and the time constant of decay of facilitation can be several tens of milliseconds (Atluri and Regehr, 1996). A role of presynaptic facilitation could therefore still be possible if $\mathrm{Ca}^{+2}$ levels quickly decrease after each presynaptic action potential. Alternatively, presynaptic enhancement of transmission might result from activity-dependent removal of tonic, autoreceptor-mediated inhibition of transmitter release (Par- nas and Parnas, 2006). At slow stimulation rates, this inhibition is maximal and synaptic transmission is suppressed. However, when presynaptic spikes occur at a sufficiently fast rate, a voltage-dependent deactivation of autoreceptors removes this brake and synaptic transmission is enhanced. Postsynaptically, superlinear summation of synaptic inputs, possibly involving NMDA-type receptors and increases in calcium conductance, might also underlie the enhancement of excitation (Polsky et al., 2004). Finally, network properties could contribute to the enhancement. For example, additional excitation to interval-counting neurons could be recruited with each successive correct interval.

The integration of excitation and inhibition in temporally specific fashions has been shown to underlie computations of signal duration (Casseday et al., 1994), response timing (Wehr and Zador, 2003), direction of spatial (Jagadeesh et al., 1993; Priebe and Ferster, 2005) or spectral (Zhang et al., 2003) motion, and, theoretically could underlie interval selectivity (Large and Crawford, 2002). Our findings reveal a new dimension in computational complexity by showing that the interplay between inhibition and PRR-dependent enhancement of excitation underlies the interval-counting properties of mid- 
brain auditory neurons; these synaptic processes contribute to the extreme selectivity of interval-counting neurons for PRR. Similarly, a rate-dependent switch from inhibition at low stimulation rates to predominantly excitation at higher stimulation rates also occurs in a hippocampal circuit (Masahiro et al., 2004). This switch involves the facilitation of excitatory synaptic transmission, as is seen for interval-counting neurons, and a depression of inhibition. It seems likely, therefore, that interplay between excitation, inhibition, and synaptic plasticity may be used for a variety of information-processing functions.

\section{References}

Alder TB, Rose GJ (1998) Long-term temporal integration in the anuran auditory system. Nat Neurosci 1:519-522.

Alder TB, Rose GJ (2000) Integration and recovery processes contribute to the temporal selectivity of neurons in the midbrain of the northern leopard frog, Rana pipiens. J Comp Physiol A Neuroethol Sens Neural Behav Physiol 186:923-937.

Andrade R, Malenka RC, Nicoll RA (1986) A G protein couples serotonin and $\mathrm{GABAB}$ receptors to the same channels in hippocampus. Science 234:1261-1265.

Atluri P, Regehr WG (1996) Determinants of the time course of facilitation at the granule cell to Purkinje cell synapse. J Neurosci 16:5661-5671.

Buonomano DV (2000) Decoding temporal information: a model based on short-term synaptic plasticity. J Neurosci 20:1129-1141.

Casseday JH, Ehrlich D, Covey E (1994) Neural tuning for sound duration: role of inhibitory mechanisms in the inferior colliculus. Science 64:847-850.

Casseday JH, Ehrlich D, Covey E (2000) Neural measurement of sound duration: control by excitatory-inhibitory interactions in the inferior colliculus. J Neurophysiol 84:1475-1487.

Cooper GW, Meyer LB (1960) The rhythmic structure of music. Chicago: University of Chicago.

Covey E, Kauer JA, Casseday JH (1996) Whole-cell patch-clamp recording reveals subthreshold sound-evoked postsynaptic currents in the inferior colliculus of awake bats. J Neurosci 16:3009-3018.

Diehl RL, Lindblom B (2004) Explaining the structure of feature and phoneme inventories: the role of auditory distinctiveness. In: Speech processing in the auditory system (Greenberg S, Ainsworth WA, Popper AN, Fay RR, eds), pp 101-162. New York: Springer.

Dittman JS, Kreitzer AC, Regehr WG (2000) Interplay between facilitation, depression, and residual calcium at three presynaptic terminals. J Neurosci 20:1374-1385.

Edwards CJ, Rose GJ (2003) Interval-integration underlies amplitude modulation band-suppression selectivity in the anuran midbrain. J Comp Physiol A Neuroethol Sens Neural Behav Physiol 189:907-914.

Edwards CJ, Alder TB, Rose GJ (2002) Auditory midbrain neurons that count. Nat Neurosci 5:934-936.

Ehret G (1996) Common rules of communication sound perception. In: Behavior and neurodynamics for auditory communication (Kanwal JS, Ehret G, eds), pp 85-114. Cambridge, UK: Cambridge UP.

Ferster D, Jagadeesh B (1992) EPSP-IPSP interactions in cat visual cortex studied with in vivo whole-cell patch recording. J Neurosci 12:1262-1274.

Gerhardt HC (2001) Acoustic communication in two groups of closely related treefrogs. Adv Study Behav 30:99-167.

Hall JC (1999) GABAergic inhibition shapes frequency tuning and modifies response properties in the midbrain of the leopard frog. J Comp Physiol A Neuroethol Sens Neural Behav Physiol 185:479-491.

Herz AVM, Gollisch T, Machens CK, Jaeger D (2006) Modeling singleneuron dynamics and computations: a balance of detail and abstraction. Science 314:80-85.

Jagadeesh B, Wheat HS, Ferster D (1993) Linearity of summation of synap- tic potentials underlying selectivity in simple cells of the cat visual cortex. Science 262:1901-1904.

Large EW, Crawford JD (2002) Auditory temporal computation: interval selectivity based on post-inhibitory rebound. J Comput Neurosci 13:125-142.

Lewicki MS (1996) Intracellular characterization of song-specific neurons in the zebra finch auditory forebrain. J Neurosci 16:5854-5863.

Lewis ER, Lombard RE (1988) The amphibian inner ear. In: The evolution of the amphibian auditory system (Fritzsch B, Ryan MJ, Wilczynski W, Hetherington TE, Walkowiak W, eds), pp 93-123. New York: Wiley.

Margoliash D, Fortune ES (1992) Temporal and harmonic combinationsensitive neurons in the zebra finch's HVc. J Neurosci 12:4309-4326.

Masahiro M, Abegg MH, Gähwiler BH, Gerber UA (2004) Frequencydependent switch from inhibition to excitation in the hippocampal unitary circuit. Nature 431:453-456.

Mooney R (2000) Different subthreshold mechanisms underlie song selectivity in identified HVc neurons of the zebra finch. J Neurosci 20:5420-5436.

Moss CF, Schnitzler H (1995) Behavioral studies of auditory information processing. In: Hearing by bats (Popper, AN, Fay RR, eds), pp 87-145. New York: Wiley.

Myrberg AA, Spanier E, Ha SJ (1978) Temporal patterning in acoustical communication. In: Contrasts in behavior (Reese ES, Lighter FJ, eds), pp 137-179. New York: Wiley.

Nelson S, Toth L, Sheth B, Sur M (1994) Orientation selectivity of cortical neurons during intracellular blockade of inhibition. Science 265:774-777.

Parnas H, Parnas I (2006) The chemical synapse goes electric: $\mathrm{Ca}^{2+}$ - and voltage-sensitive GPCRs control neurotransmitter release. Trends Neurosci 30:54-61.

Pollack GS (2001) Analysis of temporal patterns of communication signals. Curr Opin Neurobiol 11:734-738.

Polsky A, Bartlett WM, Schiller J (2004) Computational subunits in thin dendrites of pyramidal cells. Nat Neurosci 7:621-627.

Priebe NJ, Ferster D (2005) Direction selectivity of excitation and inhibition in simple cells of the cat primary visual cortex. Neuron 45:133-145.

Rose G (1986) A temporal processing mechanism for all species? Brain Behav Evol 28:134-144.

Rose GJ, Brenowitz EA (1997) Plasticity of aggressive thresholds in Hyla regilla: discrete accommodation to encounter calls. Anim Behav 53:353-361.

Rose GJ, Brenowitz EA (2002) Pacific treefrogs use temporal integration to differentiate advertisement from encounter calls. Anim Behav 63:1183-1190.

Rose G, Capranica RR (1985) Sensitivity to amplitude modulated sounds in the anuran auditory nervous system. J Neurophysiol 53:446-465.

Rose GJ, Fortune ES (1996) New techniques for making whole-cell recordings from CNS neurons in vivo. Neurosci Res 26:89-94.

Rosen MJ, Mooney R (2003) Inhibitory and excitatory mechanisms underlying auditory responses to learned vocalizations in the songbird nucleus HVC. Neuron 39:177-194.

Schmidt MF, Perkel DJ (1998) Slow synaptic inhibition in the nucleus HVc of the adult zebra finch. J Neurosci 18:895-904.

Suga N (1989) Principles of auditory information-processing derived from neuroethology. J Exp Biol 146:277-286.

Wehr M, Zador AM (2003) Balanced inhibition underlies tuning and sharpens spike timing in auditory cortex. Nature 426:442-446.

Zhang LI, Tan AYY, Schreiner CE, Merzenich MM (2003) Topography and synaptic shaping of direction selectivity in primary auditory cortex. Nature 424:201-205.

Zheng W, Hall JC (2000) GABAergic inhibition shapes frequency tuning and modifies response properties in the superior olivary nucleus of the leopard frog. J Comp Physiol 186:661-671.

Zucker RS (1999) Calcium-and activity-dependent synaptic plasticity. Curr Opin Neurobiol 9:305-313. 